\title{
Innovative Space-Time-Space Block Code for Next Generation Handheld Systems
}

\author{
Youssef Nasser and Jean-François Hélard \\ National Institute of Applied Sciences of Rennes
}

France

\section{Introduction}

Broadcasting digital TV is currently an area of intensive development and standardisation activities. Actually, different groups are working on the standardisation problem. In Europe, the digital video broadcasting (DVB) consortium has adopted different standards for terrestrial (DVB-T) fixed reception, handheld (DVB-H) reception, satellite (DVB-S) reception as well as an hybrid reception like DVB-SH. In June 2008, the DVB-T2 was born extracting a lot of specifications from DVB-S2 and proposing some specifications for an eventual use of handheld reception. Now, we are working towards a second generation of DVB-SH called next generation handheld (NGH).

Technically, DVB-SH system provides an efficient and flexible mean of carrying broadcast services over an hybrid satellite and terrestrial infrastructure operating at frequencies below $3 \mathrm{GHz}$ to a variety of portable, mobile and fixed terminals. Target terminals include handheld, vehicle-mounted, nomadic (e.g. laptops) and stationary terminals. The broadcast services encompass streaming services such as television, radio programs as well as download services enabling for example personal video recorder services. Typically, Lbands (1-2 GHz) and S-bands (2-4 GHz) are used for land mobile satellite (LMS) services. The DVB-SH system coverage is obtained by combining a satellite component (SATC) and, where necessary, a terrestrial component (TC) to ensure service continuity in areas where the satellite alone cannot provide the required quality of service (QoS). The SATC ensures wide area coverage while the TC provides cellular-type coverage. In the DVB-SH standard, two main physical layer configurations are supported. SH-A allows (but does not impose) a single frequency network (SFN) (Mattson, 2005) between the SATC and the TC, using the orthogonal frequency division multiplexing (OFDM) technique. SH-B supports a time division multiplexing (TDM) for the SATC and the OFDM technique for the TC.

The SFN presents great advantages by transmitting lower power at various sites throughout the coverage area. In an SFN, the different antennas transmit the same signal at the same moment on the same carrier frequency. The multi frequency network (MFN) allows however an optimization of the waveform and of the forward error correction (FEC) parameters according to the transmission environment. The existing SFN architectures are achieved in a single input single output system (SISO) since their deployment is very simple due to the use of one transmitting antenna by site. However, due to the increase of client services demand, it is desirable to deploy SFN with new multiple input multiple output (MIMO) techniques which ensure high spectrum efficiency as well as high diversity gain. In

Source: Communications and Networking, Book edited by: Jun Peng,

ISBN 978-953-307-114-5, pp. 434, September 2010, Sciyo, Croatia, downloaded from SCIYO.COM 
DVB-T2, the multiple input single output (MISO) technique is adopted. In the future, the combination of MIMO and OFDM techniques is pursued as a potential candidate in standardisation and proposals. In the literature, there are few studies on the SFN with MIMO transmission. (Zhang et al, 2004) proposes a new SFN model to increase the diversity gain in MIMO SFN architecture. In (Kanbe et al, 2002), an array antenna receiver using a maximum ratio combining technique is proposed to improve the system performance of the SFN transmission. The lack of studies on this original idea motivates our work to extend the application of the MIMO-OFDM transmission to the SFN architecture and NGH systems. To the authors' knowledge, no contribution has been presented on the MIMO-OFDM technique for satellite-terrestrial NGH broadcasting systems.

The aim of this work is to propose efficient MIMO schemes for SFN and for combined satellite terrestrial (SATT) transmission for NGH broadcasting systems. In this proposition, two transmission scenarios are considered: the terrestrial transmission scheme and the hybrid satellite terrestrial transmission scheme. In terrestrial transmission scheme, we investigate the possibility of applying a space-time block code (STBC) encoder between the antennas of two sites in the SFN architecture. Secondly, a generalized framework is proposed for modeling the effect of unbalanced powers received from different transmitting antennas in MIMO-OFDM systems. This is a critical problem in SFN with mobile and portable reception. Then, we analyze and compare some of the most promising STBC schemes in the context of broadcasting for future terrestrial digital TV with equal and unequal received powers. Eventually, we propose a new 3D space-time-space (STS) block code for both environments. The use of a second space dimension will be justified as being particularly adapted and efficient in the case of SFN transmission but also in hybrid SATT transmission. The proposed code is based on a double level construction of ST codes resulting from the combination of two coding schemes. The first layer corresponds to an inter-cell ST coding while the second one corresponds to an intra-cell ST coding. In hybrid transmission, the adaptation of the proposed 3D MIMO scheme is more investigated due to the difference between the satellite channel link and the terrestrial links. In our contribution, we show that the 3D MIMO scheme is efficient in line of sight (LOS) situation (with respect to the satellite) but also in low, moderate and deep shadowing situations.

\section{Functional areas addressed}

The proposition treats the problem of MIMO block in a SFN scheme. The aim of this proposition is to improve the NGH system performance and to increase its spectral efficiency by using a distributed MIMO scheme. In this solution, two schemes are proposed: $4 \times 2$ and $2 \times 2$ antenna solutions. Moreover, the proposed solutions could be applied in terrestrial transmission scheme but also could be combined with a satellite component.

\section{System model}

\subsection{System model in terrestrial SFN transmission}

Classically, in SFN architectures, the different antennas transmit at the same moment the same signal on the same frequency. In this chapter, we analyze the application of a distributed MIMO communication scheme between the antennas located at different sites of the SFN architecture. Such a system could be implemented using $M_{T}$ transmit antennas (Tx) by site as shown in Fig. 1. Without loss of generality, we will consider in our study the 
transmission behavior of two neighboring cells using a total of $\left(2 \times M_{T}\right)$ Tx and $M_{R}$ receive antennas (Rx). However, the proposed double layer scheme could be adapted to more than two neighboring cells.

From the reception viewpoint, one has to distinguish two cases: the open area and the gap area environments (Mattson, 2005) \& (Zhang et al, 2004). Open areas correspond to the areas which benefit from an unobstructed view of transmitting antennas. Gap areas correspond to the areas where the direct signal is shadowed by obstacles. In the open area case, the transmitted signal is reflected from a large number of objects in the surroundings of the receiver. These signal components are received with independently time varying amplitudes and phases. In gap areas, there is no direct signal path and the transmitted signal might be easily lost due to the obstacles. To cope with gap areas, repeaters are usually implemented in those regions. The role of these repeaters, known as gap fillers, is to adequately amplify and retransmit the signal in the gap areas. Therefore, a receiver in the gap area could operate similarly as a receiver in the open area.

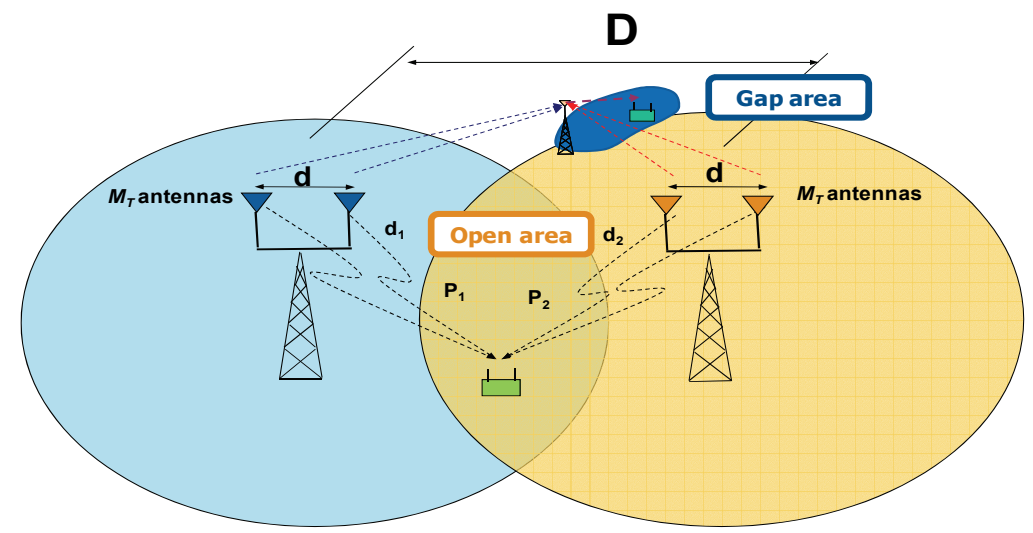

Fig. 1. SFN with unequal received powers

On the other hand, in order to make the SFN working properly the resulting total delay spread $\tau_{\max }$ of the signals received from different antennas (located at different sites) must be less than the duration of the guard interval time inserted at the beginning of each OFDM symbol. Moreover, due to the path loss, the power received by each antenna is related to this resulting delay spread as it will be shown in next subsection. Thus, the selected MIMO scheme has to deal with those transmission conditions. As a starting point, let us assume that each site holds one antenna $\left(M_{T}=1\right.$ in Fig. 1$)$ and that the receiver receives signals from both antennas. Then, two receiving environments could be presented: the open area environment and the gap area environment.

\subsubsection{Open area environment}

In the case of an open area environment, the time offset between the signals received from each site antennas could be seen as a superposition of the time offset between transmitters' signals (the signal time delay between the transmitting antennas) and the signal time offset between each transmitter and the receiver. The first offset is generally negligible since the transmitters are synchronized with an ultra stable reference like the global positioning system (GPS). The second offset could be seen as follows. When the mobile terminal (MT) 
moves within one cell, it receives signal from its own cell antenna but also from the neighboring cell antenna. Since the MT is not equidistant to both antennas, the signal received from each one will be delayed according to the position of the MT. This results into a delay $\Delta \tau$ between the two received signals from both antennas or equivalently between the channel impulse responses (CIR) between the transmitters and the receiver. The delays are directly related to the distances between the transmitters and the receiver and thus to the signal strength ratio at the receiver. Assuming an equal transmitted power $P_{0}$ at each antenna, the received power from the $i$ th antenna is:

$$
P_{i}=\frac{P_{0}}{d_{i}^{\alpha}}
$$

where $d_{i}$ is the distance between the receiver and the $i^{\text {th }}$ transmitter and $\alpha$ is the propagation constant which depends on the transmission environment.

The delay of each CIR between the $i^{\text {th }}$ transmitter and the receiver is:

$$
\tau_{i}=\frac{d_{i}}{c}
$$

where $c$ is the light celerity.

Without loss of generality, let us assume that the first transmitter site is the reference site. Substituting $d_{i}$ from (2) in (1), the CIR delay of the $i$ th link (i.e. between the $i$ th transmitter and the receiver) with respect to the reference antenna can be expressed by:

$$
\Delta \tau_{i}=\tau_{i}-\tau_{1}=\left(10^{\frac{-\beta_{i}}{10 \alpha}}-1\right) \frac{d_{1}}{c}
$$

$d_{1}$ is the distance between the reference transmitter (first one) and the receiver. $\beta_{i}$ is the received power difference (expressed in $\mathrm{dB}$ ) between the signal received from the reference site and the signal received from the $i^{\text {th }}$ transmitter. It is given by:

$$
\beta_{i}[d B]=-10 \cdot \alpha \cdot \log _{10}\left(\frac{d_{i}}{d_{1}}\right)
$$

In the sequel, we will assume that the power received from the reference antenna is equal to $0 \mathrm{~dB}$ and the distance $d_{i}$ is greater than $d_{1}$ whatever $i$. It is a real situation where the MT is closer to its own cell antenna than other antennas. In this case, $\beta_{i}$ is neither than the power attenuation factor between the $i^{\text {th }}$ transmitter and the MT. As a consequence, the transmission model becomes equivalent to a system with unbalanced powers received from each site antennas. Fig. 2 shows an example of the relation between the power attenuation factor $\beta$ and the timing offset between the CIR of the second link and the reference link in a system having two transmitting antennas located in two different sites.

If we now consider that the number of $T x$ in one site is greater than one i.e. $M_{T}>1$, the choice of an adequate MIMO scheme should then be based on this imbalance in a SFN open area environment. Moreover, it should be adequate for inter-cell (i.e. between antennas signals of each site) and intra-cell (i.e. between antennas signals in each site) environments. 


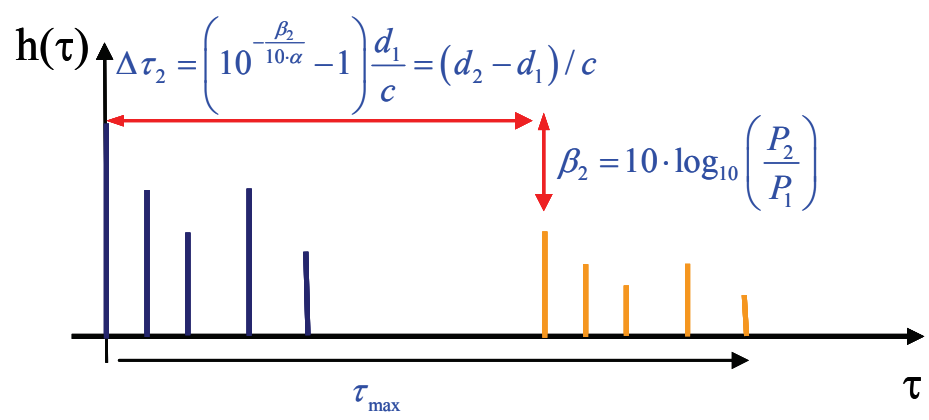

Fig. 2. Delays and powers in SFN

\subsubsection{Gap area environment}

In this case, the gap filler receives the signals from both antennas with a power difference according to equation (4). Then, it amplifies each one and retransmits them to the MT. Since the gap areas spread, in general, over a smaller region than that of the cell area, the delays between the signals received by the MT could be assumed equal to those between the signals received by the gap filler. Nevertheless, the signals received by the gap filler with a power imbalance of $\beta_{i}$ could be re-amplified with different power gains which compensate this imbalance. As a consequence, the MT could receive the different signals with the same power. In other words, due to the gap filler, the delays between the received signals from both sites' antennas could be independent of the parameters $\beta_{i}$ in the gap area environment. Thus, when designing a STBC for MIMO transmission, we should also consider this case of this terrestrial transmission scenario.

Based on this discussion on SFN transmission, it is clear that the STBC should be chosen adequately to cope with the open area and the gap area environments. This will be the subject of next sections where we propose a 3D STS code adapted for such situations.

\subsubsection{D MIMO scheme construction}

Fig. 3 depicts the transmitter modules at each site. Information bits $b_{k}$ are first channel encoded, randomly interleaved, and fed to a quadrature amplitude modulation (QAM) module. The SFN transmission system involving the two sites (described in Fig. 1) could therefore be seen as a double layer scheme in the space domain. The first layer is seen between the two sites separated by $D \mathrm{~km}$. The second layer is seen between the antennas separated by $d$ meters within one site. For the first layer, a STBC scheme is applied between the 2 signals transmitted by each site antennas. In the second layer, we use a second STBC encoder for each subset of $M_{T}$ signals transmitted from the same site. For the first layer (respectively the second layer), the STBC encoder takes $L$ (respectively $M$ ) sets of data complex symbols and transforms them into a $(2, U)$ (respectively $\left(M_{T}, V\right)$ ) output matrix according to the STBC scheme. This output is then fed to $2 \times M_{T}$ OFDM modulators, each using $N_{c}$ sub-carriers. In order to have a fair analysis and comparison between different STBC codes, the signal power at the output of the ST encoder is normalized by $2 \times M_{T}$. The double layer encoding matrix is then described by: 


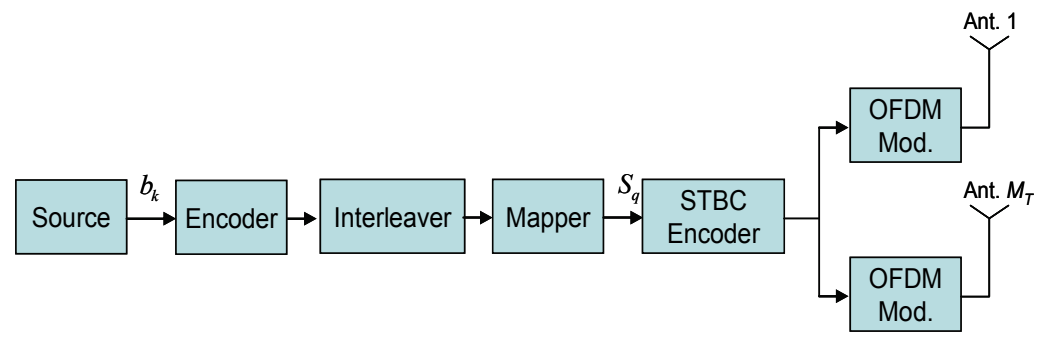

Fig. 3. MIMO-OFDM transmitter.

$$
\begin{gathered}
\mathbf{X}^{(1)}=\left(\begin{array}{ccc}
\mathbf{X}_{11}^{(2)} & \ldots & \mathbf{X}_{1 U}^{(2)} \\
\mathbf{X}_{21}^{(2)} & \ldots & \mathbf{X}_{2 U}^{(2)}
\end{array}\right) \\
\mathbf{X}_{p q}^{(2)}=\left(\begin{array}{ccc}
f_{p q, 11}\left(s_{1}, \ldots s_{M}\right) & \cdots & f_{p q, 1 V}\left(s_{1}, \ldots s_{M}\right) \\
\vdots & \ddots & \vdots \\
f_{p q, M_{T} 1}\left(s_{1}, \ldots s_{M}\right) & \cdots & f_{p q, M_{T} V}\left(s_{1}, \ldots s_{M}\right)
\end{array}\right)
\end{gathered}
$$

In (5), the superscript indicates the layer, $f_{p q, i t}\left(s_{1}, \ldots, s_{M}\right)$ is a function of the input complex symbols $s_{m}$ and depends on the STBC encoder scheme. The time dimension of the resulting $3 \mathrm{D}$ code is equal to $U \times V$ and the resulting coding rate is $R=\frac{L \times M}{U \times V}$.

In order to simplify the transmission model, the double layer encoding matrix given in (5) will be represented by $\mathbf{X}=\left[x_{i, t}\right]$ where $x_{i, t}\left(i=1, \ldots, 2 \times M_{T} ; t=1, \ldots, U \times V\right)$ is the output of the double layer STBC encoder on a given sub-carrier $n$. In other words, the layers construction is transparent from the transmission model viewpoint. Moreover, we set $Q=L \times M$ as the number of the complex symbols at the input of the double layer STBC encoder and we set $T=U \times V$ as the number of the corresponding output symbols. The ST coding rate is then $R=Q / T$.

The aim of this section is to judiciously build the proposed double layer 3D STS code so that the resulting MIMO scheme behaves efficiently in the SFN context for both aforementioned open and gap receiving environments. Then, we need to choose the adequate ST coding scheme, to apply on each layer of our 3D code.

First, we will consider the well-known orthogonal Alamouti ST coding scheme (Alamouti, 1998) for its robustness and simplicity. The Alamouti scheme provides full spatial diversity gain, no inter element interference (IEI), and requires low complexity maximum likelihood (ML) (Rupp et. al, 2004) receiver thanks to the orthogonality of its dispersion matrix. The Alamouti code has a rate $R$ equal to one and its dispersion matrix is given by:

$$
\mathbf{X}=\left[\begin{array}{cc}
s_{1} & s_{2} \\
-s_{2}^{*} & s_{1}^{*}
\end{array}\right]
$$

For non-orthogonal schemes, we consider in this work the well-known space multiplexing (SM) scheme (Foschini, 1996). SM is designed to maximize the rate by transmitting symbols sequentially on different antennas. Its coding scheme is given by:

$$
\mathbf{X}=\left[\begin{array}{ll}
s_{1} & s_{2}
\end{array}\right]^{t r}
$$


Finally, we consider the optimized Golden code (Belfiore et al, 2005) which is a full rate and fully diverse code. The Golden code is designed to maximize the rate such that the diversity gain is preserved for an increased signal constellation size. It is defined by:

$$
\mathbf{X}=\frac{1}{\sqrt{5}}\left[\begin{array}{cc}
\beta\left(s_{1}+\theta s_{2}\right) & \beta\left(s_{3}+\theta s_{4}\right) \\
\bar{\beta}\left(s_{3}+\bar{\theta} s_{4}\right) & \bar{\beta}\left(s_{1}+\bar{\theta} s_{2}\right)
\end{array}\right]
$$

where $\theta=\frac{1+\sqrt{5}}{2}, \bar{\theta}=1-\theta, \alpha=1+j(1-\theta), \bar{\alpha}=1+j(1-\bar{\theta})$.

To identify the most efficient ST code, the OFDM parameters are derived from those of the DVB-T standard (see Table 1). The spectral efficiencies 4 and 6 [b/s/Hz] are obtained for different ST schemes as shown in Table 2. In all simulations, we assume that two Rx antennas are used by the MT.

In the simulations results given hereafter, we separate the single layer case and the double layer case. For completeness point overview, we give first simulations results using a Rayleigh channel model in frequency domain i.e. we assume that the transmission from a transmitting antenna $i$ to a receiving antenna $j$ is achieved for each subcarrier $n$ through a frequency non-selective Rayleigh fading channel. The use of the i.i.d. channel model is a first approach to justify our proposed 3D STS code. For this first step, the parameters $\beta_{i}$ are chosen arbitrarily ${ }^{1}$. In a second step, we will present the results with more realistic channel model like the COST 207 TU-6 channel model (COST, 1989). In this case, the results will be given for both, open and gap area environments.

\begin{tabular}{|l|l|}
\hline FFT size & $8 \mathrm{~K}$ \\
\hline Sampling frequency $\left(f_{s}=1 / T_{s}\right)$ & $9.14 \mathrm{MHz}$ \\
\hline Guard interval $(\mathrm{GI})$ duration & $1024 \times T_{s}=112 \mu \mathrm{s}$ \\
\hline Rate $R_{c}$ of convolutional code & $1 / 2,2 / 3,3 / 4$ \\
\hline Polynomial code generator & $(133,171)_{\mathrm{o}}$ \\
\hline Channel estimation & perfect \\
\hline Constellation & $16-\mathrm{QAM}, 64-\mathrm{QAM}, 256-\mathrm{QAM}$ \\
\hline Spectral Efficiencies & $\eta=4$ and $6[\mathrm{~b} / \mathrm{s} / \mathrm{Hz}]$ \\
\hline
\end{tabular}

Table 1. Simulations Parameters

\begin{tabular}{|l|l|l|l|l|}
\hline $\begin{array}{l}\text { Spectral } \\
\text { Efficiency }\end{array}$ & ST scheme & ST rate $R$ & Constellation & $\mathrm{R}_{\mathrm{c}}$ \\
\hline \multirow{4}{*}{$\begin{array}{l}\eta=4 \\
{[\mathrm{bit} / \mathrm{Sec} / \mathrm{Hz}]}\end{array}$} & Alamouti & 1 & 64-QAM & $2 / 3$ \\
\cline { 2 - 5 } & SM & 2 & 16-QAM & $1 / 2$ \\
\cline { 2 - 5 } & Golden & 2 & 16-QAM & $1 / 2$ \\
\cline { 2 - 5 } & 3D code & 2 & 16-QAM & $1 / 2$ \\
\hline \multirow{4}{*}{$\begin{array}{l}\eta=6 \\
{[\mathrm{bit} / \mathrm{Sec} / \mathrm{Hz}]}\end{array}$} & Alamouti & 1 & 256-QAM & $3 / 4$ \\
\cline { 2 - 5 } & SM & 2 & 64-QAM & $1 / 2$ \\
\cline { 2 - 5 } & Golden & 2 & 64-QAM & $1 / 2$ \\
\cline { 2 - 5 } & 3D code & 2 & 64-QAM & $1 / 2$ \\
\hline
\end{tabular}

Table 2. Different MIMO schemes and efficiencies

1 Since we model the channel in frequency domain, there is no CIR and hence no CIR delays in this case. 


\section{a. Single Layer case: inter-cell ST coding}

The received signal at the input of the MT could be written as:

$$
\mathrm{y}=\mathrm{GBFs}+\mathrm{w}=\mathrm{G}_{\mathrm{eq}} \mathrm{s}+\mathrm{w}
$$

where the matrix $\mathbf{G}$ is composed of blocks $\mathrm{G}_{\mathrm{j}, \mathrm{i}}\left(j=1, \ldots, M_{R} ; i=1, \ldots, 2 M_{T}\right)$ each having $(2 T, 2 T)$ elements, reflecting the channel coefficients (Khalighi et al., 2006) \& (Nasser et al., 2008). B is the matrix reflecting the powers received from each antenna. $\mathbf{F}$ is composed of $2 M_{T}$ blocks of $2 T$ rows each i.e. the data transmitted on each antenna are gathered in one block having $2 T$ rows and $2 Q$ columns according to the ST coding scheme. $\mathbf{G}_{\text {eq }}$ is the equivalent channel matrix between $\mathbf{s}$ and $\mathbf{y}$. It is assumed to be known perfectly at the receiving side.

The optimal receiver is a ML (Rupp et al., 2004) receiver whose complexity increases exponentially with the number of antennas and the constellation size. In the case of orthogonal STBC (OSTBC), the optimal receiver is simply made of a concatenation of ST decoder and channel decoder modules. However, in the case of non-orthogonal STBC (NOSTBC) schemes, there is an IEI at the receiving side. The optimal receiver becomes more complex since it requires joint ST and channel decoding operations. Moreover, it requires large memory to store the different points of the trellis. In our work, we use a sub-optimal solution based on an iterative receiver where the ST detector and channel decoder exchange extrinsic information in order to enhance soft information metrics. The iterative detector shown in Fig. 4 is composed of a parallel interference canceller (PIC), a demapper which consists in computing the soft information of the transmitted bits, i.e. a log likelihood ratio (LLR) computation (Tosato \& Bisaglia, 2002), a soft-input soft-output (SISO) decoder (Hagenauer \& Hoeher,1989), and a soft mapper.

At the first iteration, the demapper takes the estimated symbols $\hat{\mathbf{s}}$, the knowledge of the channel $\mathbf{G}_{\text {eq }}$ and of the noise variance, and computes the LLR values of each of the coded bits transmitted per channel use. The estimated symbols $\hat{\mathbf{s}}$ are obtained via minimum mean square error (MMSE) filtering according to:

$$
\hat{s}_{p}^{(1)}=\mathbf{g}_{\mathbf{p}}^{\mathbf{t r}}\left(\mathbf{G}_{\mathbf{e q}} \cdot \mathbf{G}_{\mathrm{eq}}^{\mathrm{tr}}+\sigma_{w}^{2} \mathbf{I}\right)^{-1} \mathbf{y}
$$

where $\mathbf{g}_{\mathbf{p}}^{\text {tr }}$ of dimension $\left(2 M_{R} T, 1\right)$ is the $p^{\text {th }}$ column of $\mathbf{G}_{\text {eq }}(1 \leq p \leq 2 Q) . \hat{s}_{p}^{(1)}$ is the estimation of the real part ( $p$ odd) or imaginary part ( $p$ even) of $s_{q}(1 \leq q \leq Q)$. Once the estimation of the different symbols $s_{q}$ is achieved by the soft mapper at the first iteration, we use this estimation for the next iterations process.

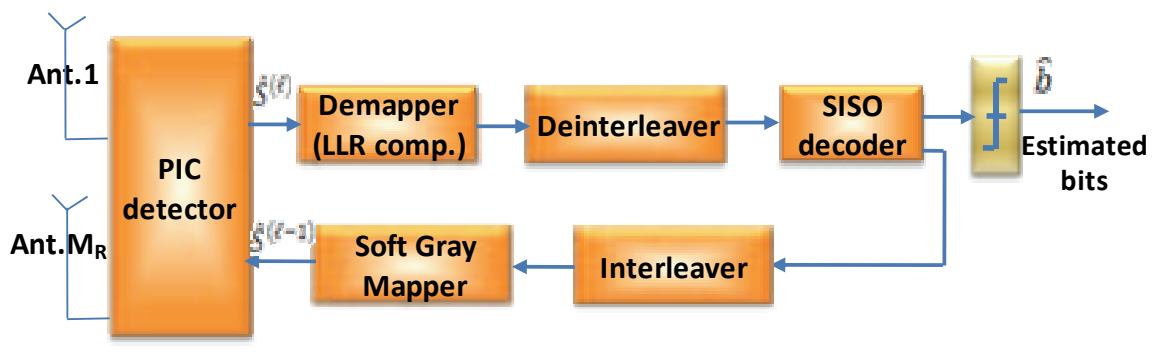

Fig. 4. Iterative receiver structure 
From the second iteration, we perform PIC operation followed by a simple inverse filtering (instead of MMSE filtering at the first iteration). This block offers enhanced LLR values to be fed to the SISO decoder by suppressing the spatial interference. This interference cancellation is described by:

$$
\begin{aligned}
& \hat{\mathbf{y}}_{\mathbf{p}}=\mathbf{y}-\mathbf{G}_{\mathbf{e q}, \mathbf{p}} \tilde{\mathbf{s}}_{\mathbf{p}}^{(\mathbf{1})} \\
& \hat{s}_{p}^{(2)}=\frac{1}{\mathbf{g}_{\mathbf{p}}^{\operatorname{tr}} \mathbf{g}_{\mathbf{p}}} \mathbf{g}_{\mathbf{p}}^{\mathbf{t r}} \hat{\mathbf{y}}_{\mathbf{p}}
\end{aligned}
$$

where $\mathbf{G}_{\mathbf{e q}, \mathbf{p}}$ of dimension $\left(2 M_{R} T, 2 Q-1\right)$ is the matrix $\mathbf{G}_{\mathbf{e q}, \mathbf{p}}$ with its $p^{\text {th }}$ column removed, $\tilde{\mathbf{s}}_{\mathbf{p}}^{(\mathbf{1})}$ of dimension $(2 Q-1,1)$ is the vector $\tilde{\mathbf{s}}$ estimated by the soft mapper with its $p^{\text {th }}$ entry removed. In our proposition, we consider a sub-optimal iterative detector for nonorthogonal schemes in order to cancel the IEI. The iterative process used here converges after 3 iterations (Nasser et al., May 2008). Therefore, all the results given thereafter are obtained after 3 iterations.

In the case of single layer reception, we have one antenna by site. Then, the second layer matrix $\mathbf{X}^{(2)}$ in (5) resumes to one element. The multiple input component of the MIMO scheme is then only obtained by the single antenna in each site $\left(M_{T}=1\right)$. Due to the mobility, the MT is assumed to occupy different locations and the first layer ST scheme must be efficient face to unequal received powers. For equal received powers, we assume that the powers of the matrix $\mathbf{B}$ in (9) are equal to $0 \mathrm{~dB}$.

Fig. 5 gives the required $E_{b} / N_{0}$ to obtain a $B E R=10^{-4}$ for a spectral efficiency $\eta=4[b / s / H z]$. Moreover, since we have one Tx antenna by site, we set $\beta_{1}=0 \mathrm{~dB}$ and we change $\beta=\beta_{2}$. As expected, this figure shows that the Golden code presents the best performance when the $\mathrm{Rx}$ receives the same power from both sites (i.e. $\beta_{1}=\beta_{2}=0 \mathrm{~dB}$ ). When $\beta_{2}$ decreases, the Alamouti scheme is very efficient and presents a maximum loss of only $3 \mathrm{~dB}$ in terms of required $\mathrm{E}_{\mathrm{b}} / \mathrm{N}_{0}$ with respect to equal received powers case. Indeed, for very small values of $\beta$, the

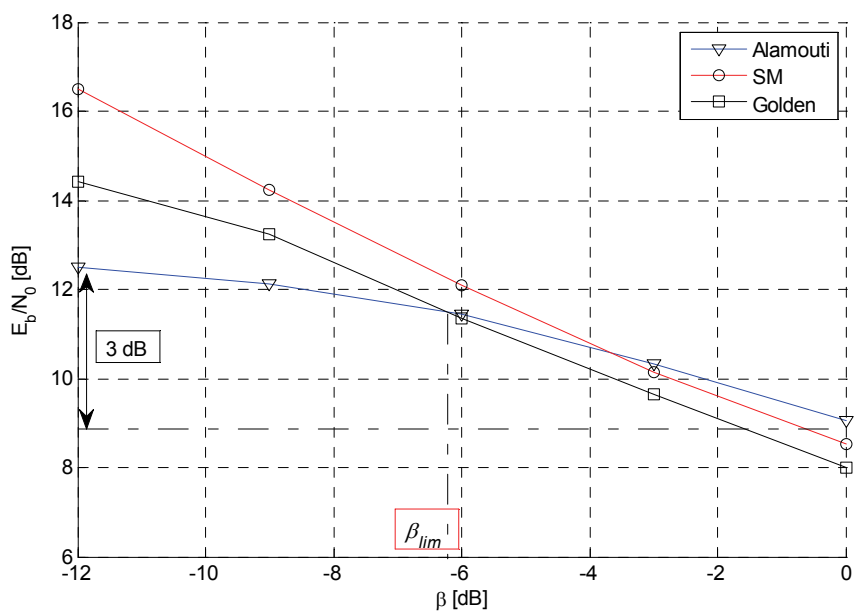

Fig. 5. Required Eb/N0 to obtain a $B E R=10^{-4}$, single layer case, $\eta=4[b / s / H z]$ 


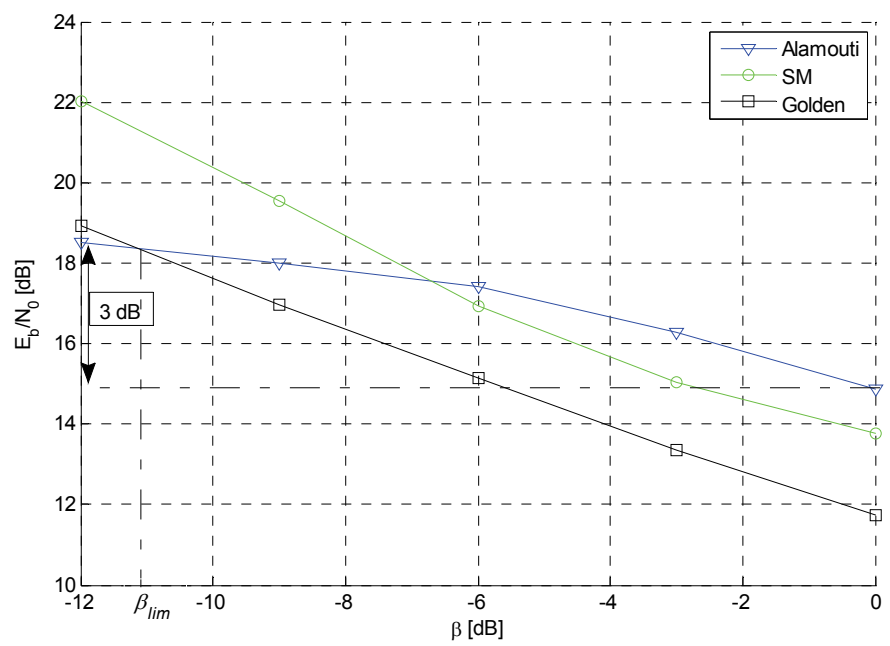

Fig. 6. Required Eb/N0 to obtain a BER=10-4, single layer case, $\eta=4[b / s / H z]$

transmission scenario becomes equivalent to a transmission scenario with one transmitting antenna. In this figure, the value $\beta_{\text {lim }}=-6.2 \mathrm{~dB}$ presents the power imbalance limit where the Alamouti and the Golden code schemes have the same performance at a BER=10-4.

The same kind of results, shown in Fig. 6, are observed for a spectral efficiency $\eta=6$ $[\mathrm{b} / \mathrm{s} / \mathrm{Hz}]$. The Alamouti scheme is more efficient than the Golden code when the power imbalance parameter $\beta$ becomes less than a given limit value $\beta_{\text {lim }}=-11.2 \mathrm{~dB}$.

b. Double Layer case: intra-cell ST coding

Considering the whole double layer space domain construction, one ST coding scheme has to be assigned to each layer of the proposed system. The resulting 3D STS code should be efficient for both environments in SFN architecture. We propose to construct the first layer, i.e. the inter-cell coding, with Alamouti scheme since it is the most resistant for the unequal received powers case. In a complementary way, we propose to construct the second layer, i.e. the intra-cell coding, with the Golden code since it offers the best results in the case of equal received powers. After combination of the two space layers with time dimension, (5) yields:

$$
X=1 / \sqrt{5}\left(\begin{array}{cccc}
\alpha\left(s_{1}+\theta s_{2}\right) & \alpha\left(s_{3}+\theta s_{4}\right) & \alpha\left(s_{5}+\theta s_{6}\right) & \alpha\left(s_{7}+\theta s_{8}\right) \\
j \bar{\alpha}\left(s_{3}+\bar{\theta} s_{4}\right) & \bar{\alpha}\left(s_{1}+\bar{\theta} s_{2}\right) & j \bar{\alpha}\left(s_{7}+\bar{\theta} s_{8}\right) & \bar{\alpha}\left(s_{5}+\bar{\theta} s_{6}\right) \\
-\alpha^{*}\left(s_{5}^{*}+\theta^{*} s_{6}^{*}\right) & -\alpha^{*}\left(s_{7}^{*}+\theta^{*} s_{8}^{*}\right) & \alpha^{*}\left(s_{1}^{*}+\theta^{*} s_{2}^{*}\right) & \alpha^{*}\left(s_{3}^{*}+\theta^{*} s_{4}^{*}\right) \\
\overline{j \alpha^{*}}\left(s_{7}^{*}+\overline{\theta^{*}} s_{8}^{*}\right) & -\overline{\alpha^{*}}\left(s_{5}^{*}+\overline{\theta^{*}} s_{6}^{*}\right) & -j \alpha^{*}\left(s_{3}^{*}+\overline{\theta^{*}} s_{4}^{*}\right) & \overline{\alpha^{*}}\left(s_{1}^{*}+\overline{\theta^{*}} s_{2}^{*}\right)
\end{array}\right)
$$

where $\theta=\frac{1+\sqrt{5}}{2}, \bar{\theta}=1-\theta, \alpha=1+j(1-\theta), \bar{\alpha}=1+j(1-\bar{\theta})$.

\subsubsection{Simulation results in open area environment}

In an open area environment, the MT is in an unobstructed region with respect to each site antennas. Since the distance $d$ between the transmitting antennas in one site is negligible 
with respect to the distance $D$ (Fig. 1), the power attenuation factors in the case of our 3D code are such that $\beta_{1}=\beta_{2}=0 \mathrm{~dB}$ and $\beta=\beta_{3}=\beta_{4}$.

Fig. 7 shows the results in terms of required $E_{b} / N_{0}$ to obtain a BER equal to $10^{-4}$ for different values of $\beta$ and 3 STBC schemes i.e. our proposed 3D code scheme, the single layer Alamouti and the Golden code schemes assuming Rayleigh i.i.d frequency channel coefficients. In this figure, the value $\beta$ corresponds to $\beta_{2}$ for the single layer case and to $\beta=\beta_{3}=\beta_{4}$ for our 3D code. Fig. 7 shows that the proposed scheme presents the best performance whatever the spectral efficiency and the factor $\beta$ are. Indeed, it is optimized for SFN systems and unbalanced received powers. For $\beta=-12 \mathrm{~dB}$, the proposed 3D code offers a gain equal to $1.8 \mathrm{~dB}$ (respectively $3 \mathrm{~dB}$ ) with respect to the Alamouti scheme for a spectral efficiency $\eta=4[\mathrm{~b} / \mathrm{s} / \mathrm{Hz}]$ (resp. $\eta=6$ $[\mathrm{b} / \mathrm{s} / \mathrm{Hz}])$. This gain is greater when it is compared to the Golden code. Moreover, the maximum loss of our code due to unbalanced received powers is equal to $3 \mathrm{~dB}$ in terms of $\mathrm{E}_{\mathrm{b}} / \mathrm{N}_{0}$. This means that it leads to a powerful code for SFN systems.

In a MIMO COST 207 TU-6 channel model, we assume that the MT is moving with a velocity of $10 \mathrm{~km} / \mathrm{h}$ and the distance $d_{1}$ between the receiver and the reference antenna is equal to 5 $\mathrm{km}$. The CIRs between different transmitters and the MT are delayed according to (3).

Fig. 8 gives the same kind of results of those given in Fig. 7. Once again, these results highlight the superiority of the proposed $3 \mathrm{D}$ code in real channel models whatever the spectral efficiency and the factor $\beta$ i.e. the $3 \mathrm{D}$ code outperforms the others schemes in all cases. The gain could reach $1.5 \mathrm{~dB}$ for a spectral efficiency $\eta=4[\mathrm{~b} / \mathrm{s} / \mathrm{Hz}]$ and $3.1 \mathrm{~dB}$ for a spectral efficiency $\eta=6[\mathrm{~b} / \mathrm{s} / \mathrm{Hz}]$.

Fig. 9 evaluates the robustness of the different schemes to the MT velocity. We assume that the MT is moving within one cell with a velocity of $10 \mathrm{~km} / \mathrm{h}$ and $60 \mathrm{~km} / \mathrm{h}$ respectively. We show in this figure that the Alamouti scheme is very robust to the MT velocity. The degradation of the Golden code might reach $1 \mathrm{~dB}$ in terms of required $\mathrm{Eb} / \mathrm{N} 0$ to reach a $\mathrm{BER}=10^{-4}$. The degradation of the proposed $3 \mathrm{D}$ code due to the MT velocity and hence to the Doppler effect is of about $0.2 \mathrm{~dB}$ only.

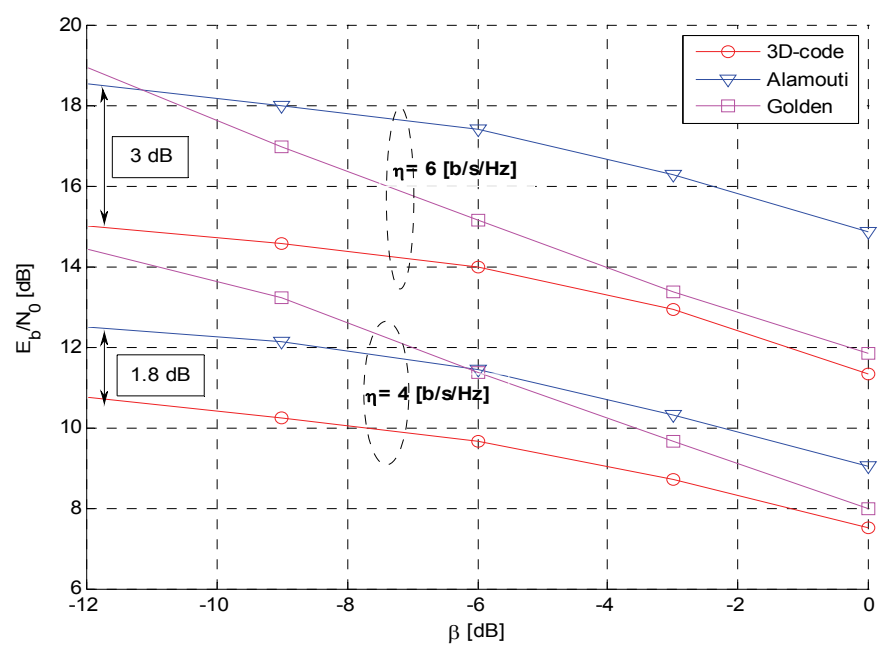

Fig. 7. Required Eb/N0 to obtain a BER=10-4, double layer case, $\eta=4[b / s / H z], \eta=6$ $[\mathrm{b} / \mathrm{s} / \mathrm{Hz}]$, Rayleigh channel 


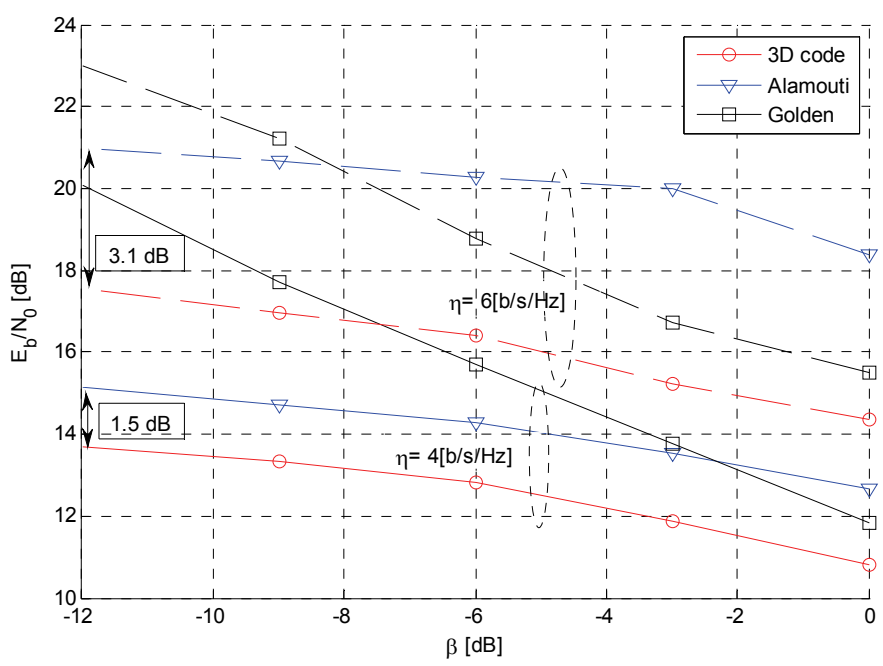

Fig. 8. Required $E_{b} / N_{0}$ to obtain a $B E R=10^{-4}$, double layer case, $\eta=4[b / s / H z], \eta=6[b / s / H z]$, TU-6 channel

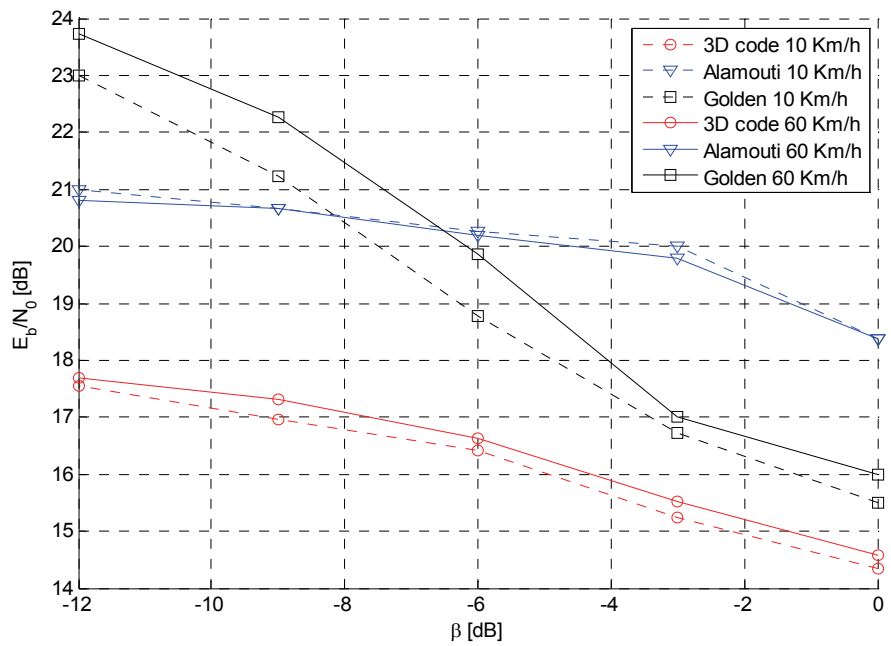

Fig. 9. Required $E_{b} / N_{0}$ to obtain a $B E R=10^{-4}, \eta=6[b / s / H z], T U-6$ channel, different values of MT velocity

\subsubsection{Simulation results in gap area environment}

In a gap area environment, the MT is in obstruction with respect to each site antennas. In this case, the gap filler receiving antennas become at the same situation of those of the MT in the open area environment i.e. a power imbalance is observed at the receiving side and it is related to the CIR delays by equation (4). However, due to the gap filler amplification, the power received by the MT in a gap area could be independent of these delays. 


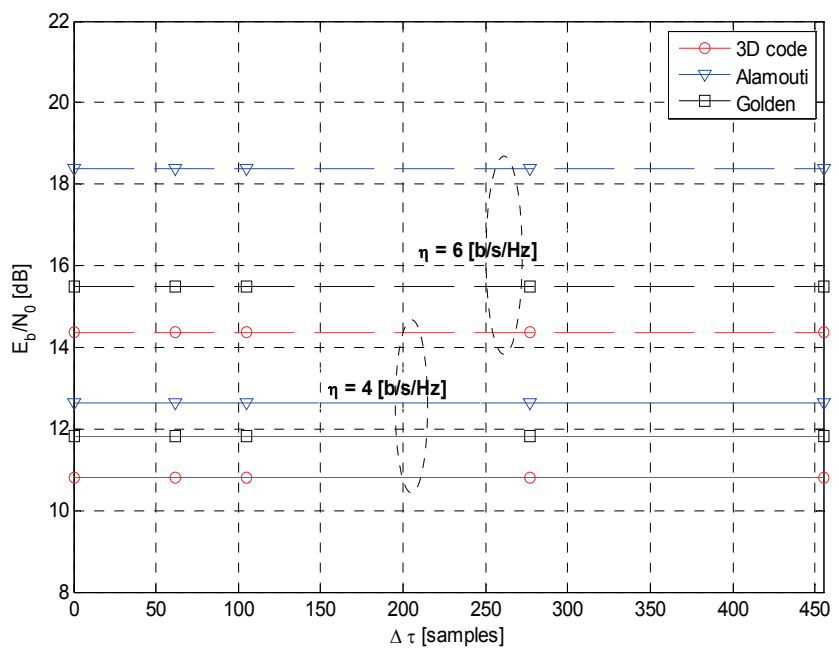

Fig. 10. Required $E_{b} / N_{0}$ to obtain a $B E R=10^{-4}, \eta=4[b / s / H z], \eta=6[b / s / H z], T U-6$ channel, gap area environment.

In Fig. 10, we give the required $\mathrm{E}_{\mathrm{b}} / \mathrm{N}_{0}$ that a MT needs in a gap area to obtain a BER $=10^{-4}$ with respect to the CIR delays $\Delta \tau$ observed at the gap filler receivers. As expected, we show in this figure that the results are independent of these delays since they are smaller than the guard interval durations ( $\mathrm{GI}=1024$ samples). In other words, as these delays are less than the guard interval duration, they produce only a phase rotation which is corrected by the equalizer in the frequency domain. The power imbalance is already corrected by the gap filler amplification.

\subsection{System model in hybrid satellite terrestrial transmission}

For hybrid SATT transmission, we propose to apply the MIMO scheme between the terrestrial and satellite sites as described in Fig. 11. Due to the links model difference, i.e. satellite link and terrestrial link, the proposed code has to cope with different transmission scenarios. More precisely, the MIMO scheme has to be efficient in the LOS region but also in shadowing regions (moderate and deep) with respect to the satellite antennas. In order to achieve that, we propose again to use the 3D MIMO scheme for such situations. The first layer corresponds to the inter-cell ST coding, i.e. between satellite and terrestrial antennas, while the second corresponds to the intra-cell ST coding, i.e. between the antennas of the same site. For the satellite links, we have considered the land mobile satellite (LMS) (Murr et al., 1995) adopted in DVB-SH (ETSI, 2008) and described by Fontan (Fontan et al., 2001), (Loo, 1985) \& (Fontan et al., 1998). The LMS channel is modeled by Markov chain with three states. The state S1 corresponds to the LOS situation, while S2 and S3 correspond respectively to the moderate and deep shadowing situations. Generally speaking, the LMS channel in each state follows a Loo distribution (Loo, 1985). The latter is a Rice distribution where its mean follows a log-normal distribution having a mean $\mu$ and a standard deviation $\Sigma$. Table 3 shows that the different states of the Markov chain depend on the elevation angles and that each state has its specified mean and standard deviation. The parameter MP in this table reflects the multipath component power in the Rice distribution. 


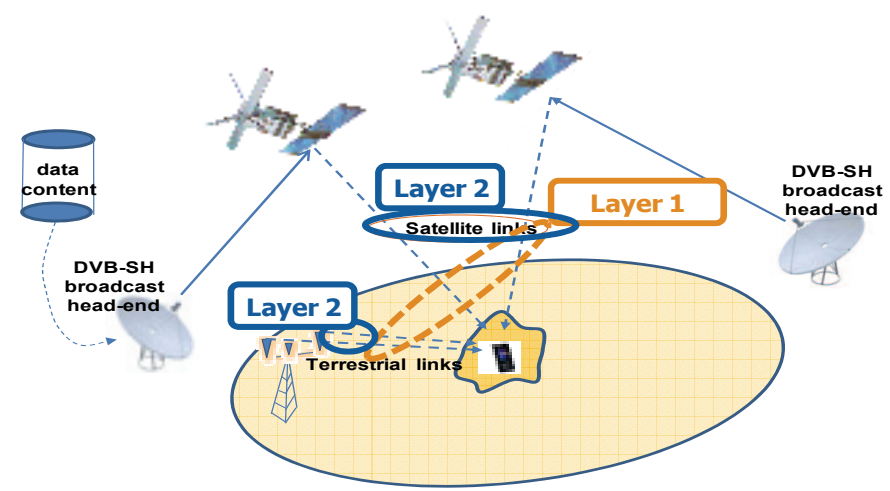

Fig. 11. Layered STS 3D code using SATT transmission scheme

\subsubsection{First layer construction: SATT coding}

In order to construct the first layer, we consider the same method as done for terrestrial transmission. First, we will construct the first layer using the well-known MIMO schemes, i.e. Alamouti and Golden codes. Second, due to the mobility, the MT is assumed to occupy different locations over a sufficient long route. Then, the first layer ST scheme must be efficient face to shadowing during its trajectory. Recall that the moderate and deep shadowing are dependent of the elevation angle. For example, in Table 3, the moderate shadowing for an elevation angle of $30^{\circ}$ corresponds to a mean value $\mu=-4.7 \mathrm{~dB}$ and the deep shadowing corresponds to a mean value equal to $-7 \mathrm{~dB}$. It is clear from Table 3 that for an elevation angle equal to $30^{\circ}$, the system presents the highest signal power level since the moderate and deep shadowing are relatively acceptable comparing to other elevation angles $\theta$. In the sequel, we will present first the results obtained with an elevation angle $\theta=30^{\circ}$ and $\theta=50^{\circ}$ and for the various spectral efficiencies using an Alamouti and Golden code scheme at the first layer. Fig. 12 shows the required $E_{b} / N_{0}$ to obtain a BER equal to $10^{-4}$ for a spectral efficiency $\eta=2,4$ and $6 \mathrm{~b} / \mathrm{s} / \mathrm{Hz}$. As expected, we conclude from these results that for low spectral efficiency, i.e. $\eta=2$, the Alamouti scheme outperforms the Golden scheme. However, for a spectral efficiency $\eta=4$ and $\eta=6$, the conclusion on the best performance is not immediate. It depends on the elevation angle and hence on the shadowing level. For high shadowing level (see Table $3, \theta=50^{\circ}$ ), the Alamouti code presents almost better

\begin{tabular}{l|c|c|c|c|c|c|c|c|c}
\hline \hline \multirow{3}{*}{ Elevation } & \multicolumn{3}{|c|}{ S1: LOS } & \multicolumn{3}{c|}{$\begin{array}{c}\text { S2: Interm. } \\
\text { Shadowing }\end{array}$} & \multicolumn{3}{c}{$\begin{array}{c}\text { S3: Deep } \\
\text { Shadowing }\end{array}$} \\
\cline { 2 - 10 } & $\mu$ & $\Sigma$ & MP & $\mu$ & $\Sigma$ & MP & $\mu$ & $\Sigma$ & MP \\
\hline \hline $10^{\circ}$ & -0.1 & 0.5 & -19 & -8.7 & 3 & -12 & -12.1 & 6 & -25 \\
$30^{\circ}$ & -0.5 & 1 & -15 & -4.7 & 1.5 & -19 & -7 & 3 & -20 \\
$50^{\circ}$ & -0.5 & 1 & -17 & -6.5 & 2.5 & -17 & -14 & 2.5 & -20 \\
$70^{\circ}$ & -0.2 & 0.5 & -15 & -6.0 & 2.1 & -17 & -11.5 & 2 & -20 \\
\hline \hline
\end{tabular}

Table 3. Average Loo model parameters in $\mathrm{dB}$ for various angles and suburban area (measurement results given in (Fontan et al., 1985)) 


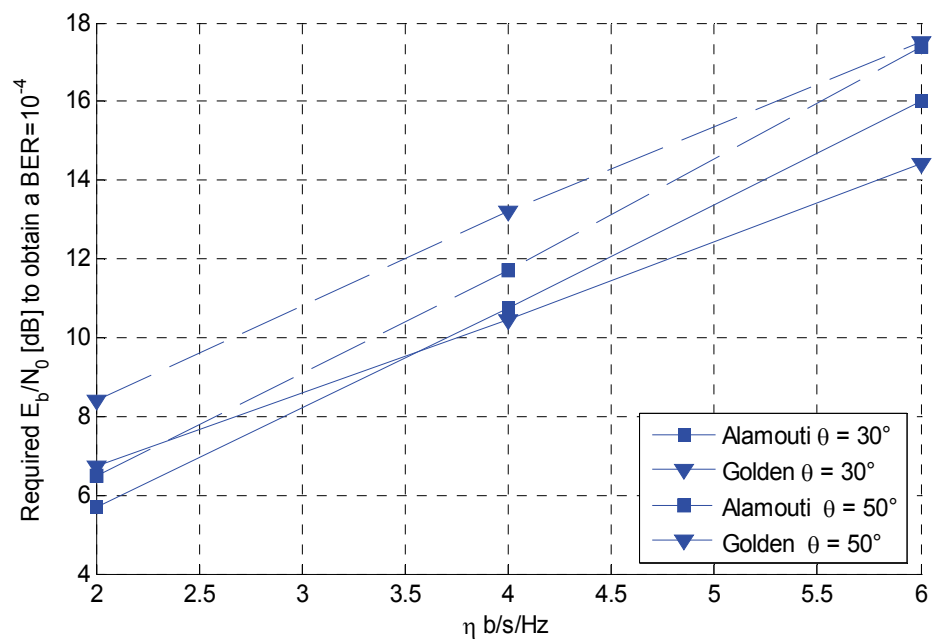

Fig. 12. Required $E_{b} / N_{0}$ to obtain a $B E R=10^{-4}$, single layer case

performance. In summary, the Golden code scheme outperforms the Alamouti scheme only for high spectral efficiency and relatively low shadowing levels $\left(\theta=30^{\circ}\right)$. This confirms our results in terrestrial transmission where the performance of the MIMO scheme depends on the power imbalance between the two signals received from each site.

\subsubsection{Second layer construction: intra-site coding}

Considering the whole layers' construction (i.e. $M_{T}>1$ ), one ST coding scheme has to be assigned to the SATT coding and another ST coding scheme has to be assigned to the intrasite coding. The resulting layered ST coding should be efficient for low, moderate and deep shadowing levels. Considering the results and conclusions obtained in previous sub-section, we propose to construct the SATT layer with Alamouti scheme, since it is the most resistant for the deep shadowing levels. In a complementary way, we propose to construct the second layer with the Golden code since it offers the best results in the case of relatively low shadowing levels.

Fig. 13 shows the results in terms of required $E_{b} / N_{0}$ to obtain a BER equal to $10^{-4}$ for the various elevation angles, two spectral efficiencies $\eta=2 \mathrm{~b} / \mathrm{s} / \mathrm{Hz}$ and $\eta=6 \mathrm{~b} / \mathrm{s} / \mathrm{Hz}$ and the three considered codes i.e. our proposed 3D scheme, the single layer Alamouti scheme and the single layer Golden scheme. The results obtained in this figure show that the proposed 3D scheme outperforms the other schemes whatever the elevation angle and the spectral efficiency are. Moreover, as expected, the best performance is obtained for an elevation angle $\theta=30^{\circ}$. The gain of the 3D code compared to the Alamouti scheme is about $1 \mathrm{~dB}$ for $\eta=2 \mathrm{~b} / \mathrm{s} / \mathrm{Hz}$ and can reach $4 \mathrm{~dB}$ for $\eta=6 \mathrm{~b} / \mathrm{s} / \mathrm{Hz}$. The conclusions of Fig. 13 are confirmed in Fig. 14 for $\eta=4 \mathrm{~b} / \mathrm{s} / \mathrm{Hz}$. This means that the 3D code leads to a powerful code for next DVB-NGH systems.

\subsection{Conclusions}

In this work, we have presented a full rate full diversity 3D code, a promising candidate for next generation broadcast technologies. It is constructed using two layers: the first layer 
using Alamouti code and the second layer using Golden code. We showed that our proposed scheme is very efficient to cope with low, moderate and deep shadowing levels as well as various elevation angles. The proposed scheme is fully compatible with SFN and hybrid SATT scheme. It is then a very promising candidate for the broadcasting of the future terrestrial digital TV through NGH structures.

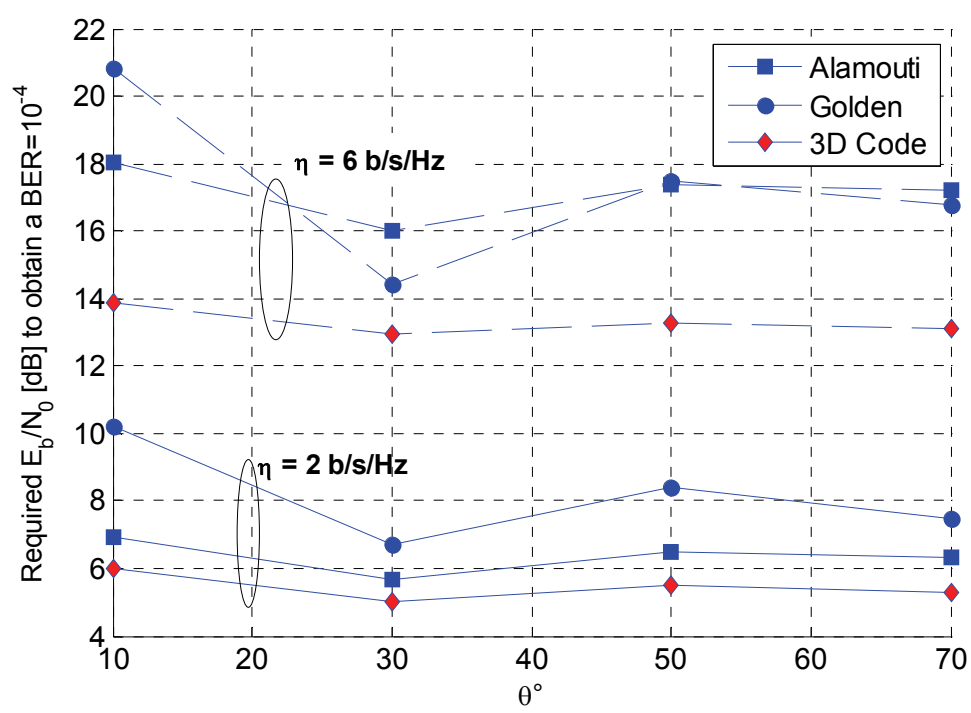

Fig. 13. Required $E_{b} / N_{0}$ to obtain a $B E R=10^{-4}$, double layer construction, $\eta$ variable

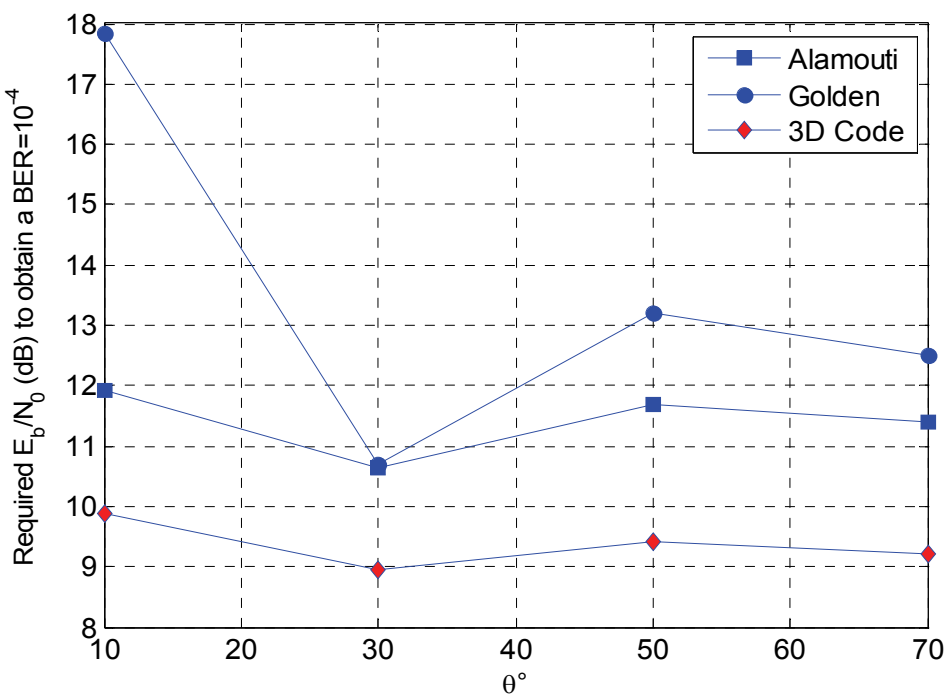

Fig. 14. Required $E_{b} / N_{0}$ to obtain a $B E R=10^{-4}$, double layer construction, $\eta=4 \mathrm{~b} / \mathrm{s} / \mathrm{Hz}$ 


\section{References}

Mattson A. (2005), Single frequency networks in DTV, IEEE Trans. on Broadcasting, Vol. 51, Issue 4, Dec. 2005, pp. 413-422, ISSN : 0018-9316.

Zhang L., Gui L., Qiao Y., and Zhang W. (2004), Obtaining diversity gain for DTV by using MIMO structure in SFN, IEEE Trans. on broadcasting, Vol. 50, No. 1, March 2004, 8390, ISSN: 0018-9316.

Kanbe Y., Itami M., Itoh K., and Aghvami A. (2002), Reception of an OFDM signal with an array antenna in a SFN environment, Proc. of IEEE Personal Indoor and Mobile Radio Communications, Vol. 3, 1310-1315, ISBN: 0-7803-7589-0, Sept. 2002.

Alamouti, S.M. (1998), A simple transmit diversity technique for wireless communications, IEEE Journal on Selected Areas in Communications, Vol. 16, No. 8, Oct. 1998, 14511458, ISSN: 0733-8716.

Rupp M., Gritsh G., Weinrichter H. (2004), Approximate ML detection for MIMO systems with very low complexity, Proc. of the International conference on Acoustics, Speech, and Signal Processing, Vol. 4, pp. 809-812, ISBN: 0-7803-8484-9, May 2004.

Foschini G. J. (1996), Layered space-time architecture for wireless communication in a fading environment when using multi-element antenna, Bell Labs Tech. Journal, Vol. 1, no. 2, 41-59.

Belfiore, J.-C., Rekaya G., \& Viterbo E. (2005). The golden code: a $2 \times 2$ full-rate space-time code with non vanishing determinants, IEEE Transactions in Information Theory, Vol. 51, No. 4, April 2005, 1432-1436, ISSN : 0018-9448.

COST (1989), COST 207 Report, Digital Land Mobile Radio Communications, Commission of European Communities, Directorate General, Telecommunications Information Industries and Innovation, Luxemburg.

Khalighi M. A., Hélard J.-F., and Bourennane S. (2006), Contrasting Orthogonal and non orthogonal space-time schemes for perfectly-known and estimated MIMO channels, Proc. of IEEE Int. Conf. on Communications systems, 1-5, ISBN: 1-4244-04118, Oct. 2006, Singapore.

Nasser, Y.; Helard, J.-F. \& Crussiere, M. (2008). System Level Evaluation of Innovative Coded MIMO-OFDM Systems for Broadcasting Digital TV. International Journal of Digital Multimedia Broadcasting, Vol. 2008, pages 12, doi:10.1155/2008/359206.

Nasser Y., Hélard J.-F., Crussiere M., and Pasquero O. (2008), Efficient MIMO-OFDM schemes for future terrestrial digital TV with unequal received powers, Proc. of IEEE International Communications Conference, 2021 - 2027, ISBN: 978-1-4244-2075-9, June 2008, Bejing, China.

Tosato F., and Bisaglia P. (2002), Simplified Soft-Output Demapper for Binary Interleaved COFDM with Application to HIPERLAN/2, Proc IEEE Int. Conf. on Communications, pp. 664-668, ISBN: 0-7803-7400-2, June 2002.

Hagenauer J., and Hoeher P. (1989), A Viterbi algorithm with soft-decision outputs and its applications, Proc. of IEEE Global Telecommunications Conf., pp. 1680-1686, Nov. 1989, Dallas, USA.

Murr F., Kastner-Puschl S., Bolzano B., Kubista E. (1995), Land mobile Satellite narrowband propagation measurement campaign at Ka-Band, ESTEC contract 9949/92NL, Final report.

ETSI (2008). DVB-SH Implementation Guidelines. TM-SSP252r9f. 
Fontan F., Vazquez-Castro M., Cabado C., Garcia J., Kubista E. (2001), Statistical modeling of the LMS channel, IEEE Trans. on Vehicular Technology, Vol. 50, No.6, Nov. 2001, 1549-1567, ISSN: 0018-9545.

Loo C. (1985), A Statistical Model for a Land Mobile Satellite Link, IEEE Trans. Vehicular. Technology, Vol. VT-34, No.3, August 1985, 122-127, ISSN: 0018-9545.

Fontan F., Vazquez-Castro M., Buonomo S., Baptista P., and Arbesser-Rastburg B. (1998), SBand LMS propagation channel behavior for different environments, degrees of shadowing and elevation angles, IEEE Trans. on Broadcasting, Vol. 44, March 1998, 40-76, ISSN: 0018-9316.

Loo C. (1991), Further results on the statistics of propagation data at L-band (1542 MHz) for mobile satellite communications, Proc. of IEEE Vehicular Technology Conference, pp. 51-56, ISSN: 1090-3038, May 1991, Saint Louis, USA. 


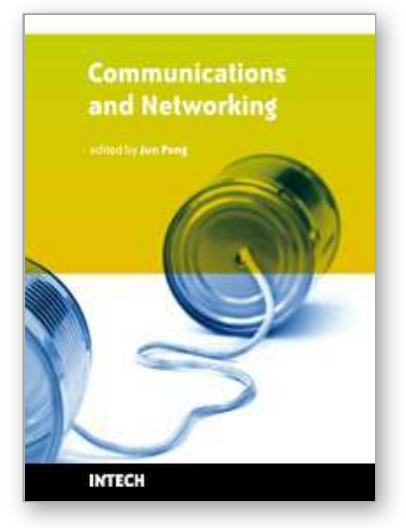

\section{Communications and Networking \\ Edited by Jun Peng}

ISBN 978-953-307-114-5

Hard cover, 434 pages

Publisher Sciyo

Published online 28, September, 2010

Published in print edition September, 2010

This book "Communications and Networking" focuses on the issues at the lowest two layers of communications and networking and provides recent research results on some of these issues. In particular, it first introduces recent research results on many important issues at the physical layer and data link layer of communications and networking and then briefly shows some results on some other important topics such as security and the application of wireless networks. In summary, this book covers a wide range of interesting topics of communications and networking. The introductions, data, and references in this book will help the readers know more abut this topic and help them explore this exciting and fast-evolving field.

\section{How to reference}

In order to correctly reference this scholarly work, feel free to copy and paste the following:

Youssef Nasser and Jean-François Helard (2010). Innovative Space-Time-Space Block Code for Next Generation Handheld Systems, Communications and Networking, Jun Peng (Ed.), ISBN: 978-953-307-114-5, InTech, Available from: http://www.intechopen.com/books/communications-and-networking/innovative-spacetime-space-block-code-for-next-generation-handheld-systems

\section{INTECH}

open science | open minds

\section{InTech Europe}

University Campus STeP Ri Slavka Krautzeka 83/A 51000 Rijeka, Croatia Phone: +385 (51) 770447

Fax: +385 (51) 686166 www.intechopen.com

\section{InTech China}

Unit 405, Office Block, Hotel Equatorial Shanghai No.65, Yan An Road (West), Shanghai, 200040, China 中国上海市延安西路65号上海国际贵都大饭店办公楼405单元 Phone: +86-21-62489820

Fax: +86-21-62489821 
(C) 2010 The Author(s). Licensee IntechOpen. This chapter is distributed under the terms of the Creative Commons Attribution-NonCommercialShareAlike-3.0 License, which permits use, distribution and reproduction for non-commercial purposes, provided the original is properly cited and derivative works building on this content are distributed under the same license. 